\title{
Clinical trials: the muddled Canadian landscape
}

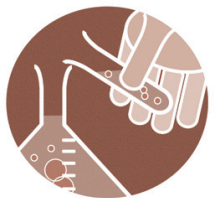

This overview of the landscape of Canadian clinical trials, along with a historical primer (page 23), is the first in a series of articles CMAJ will present this year on the state of clinical trials in Canada and abroad. Many believe the system is in disarray because of spiralling costs and conflicting requirements. Among the issues that will be explored in upcoming articles are costs, recruitment, registration, ethical oversight, patient safety, reporting and the push for reforms.

I ndustry, government and academia will have to collaborate if Canada is to maintain or increase its share of clinical trials in the highly competitive global market, speakers told the fifth annual Clinical Trials in Canada conference.

"We think there is a crisis of clinical trials leaving Canada and there is fear that once they are gone they will be hard to get back," said Ronald Heslegrave, chair of the ethics board at the University Health Network in Toronto, Ontario.

Compounding the problem is the difficulty in recruiting physicians to help run trials. "Few doctors in their 30 s and 40s are interested in clinical trials or want to be trialists," said Dr. Mary Bell, head of the rheumatology division at the Sunnybrook Health Sciences Centres in Toronto.

"We need to think about succession. We need to be more organized about training for subinvestigators," while some current investigators need retraining, she told the conference, held Oct. 21 and 22 in Toronto.

Canada has experienced an increase in the number of phase I trials - a reflection of a Health Canada move to shorten the time to review these trials and a significant increase in spending worldwide at the phase I trial level. But the numbers of phase II and III trials have levelled or declined in the past few years (Fig. 1).

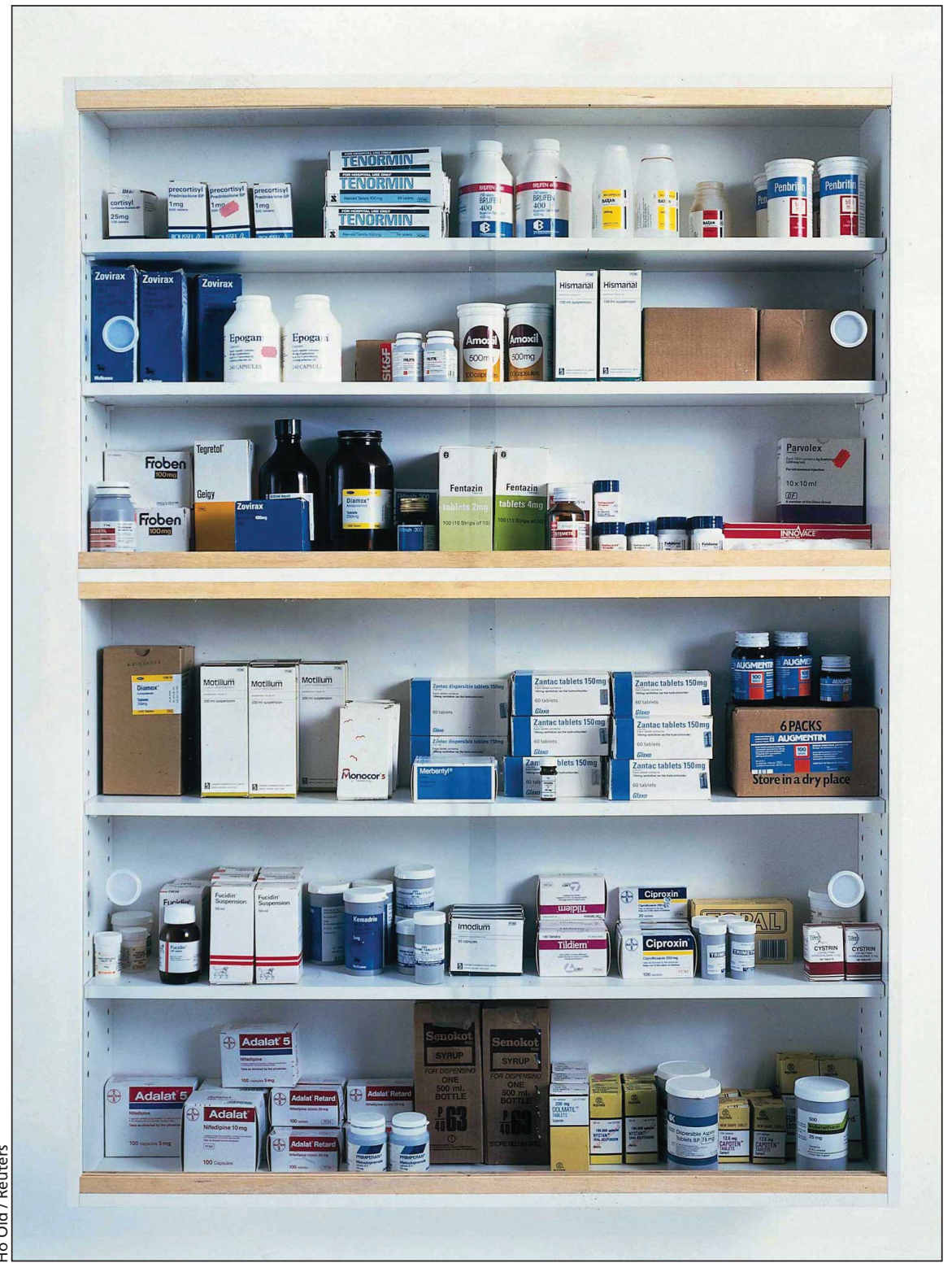

This artwork, entitled "God," fetched $\mathrm{f} 188500$ (about Can $\$ 353$ 670) for artist Damien Hirst at auction. Medicine and drugs are recurring themes in this British artist's work.

"My sense is it would be a success just to maintain the current level of research and development," commented Pierre Gervais, president of Q\&T Research in Sherbrooke, Quebec.

Pharmaceutical companies are the main sponsors of clinical trials in Canada. In 2007, spending on clinical trials (close to $\$ 600$ million) accounted for $78 \%$ of the companies' applied research expenditures.

The vast majority $(88.7 \%)$ of the companies' research and development spending was in Ontario and Quebec, according to the Patented Medicines Prices Review Board's 2007 annual report.

Pharmaceutical companies are increasingly outsourcing the running of 
clinical trials to contract research organizations, which organize trials both in the community and within hospitals.

Most phase I trials, however, are conducted by specialized contract research organizations, said Brett Wilson, an associate director of clinical site monitoring for Bristol-Myers Squibb Canada.

Fifteen to 20 years ago "Canada was the new kid" in clinical trials, but with $4.1 \%$ of clinical trial sites, Canada now ranks fourth in the world, after the United States (48.7\%), Germany $(5.7 \%)$ and France $(4.3 \%)$, he said, citing data from a recent article on the globalization of clinical trials (Nature Reviews Drug Discov 2008;[7]:13-4).

But the frontrunners are being challenged by the rapid expansion of capacity in countries such as Russia, Argentina, India, Hungary and China.

Canada's clinical trials future will depend on cultivating the expertise to run smaller, more sophisticated trials, speakers from academic centres argued. "Trials looking at small effects and requiring large numbers of patients will not be done in Canada. ... The focus will be targeted drugs producing bigger effects in smaller populations," said Robert Phillips, deputy director of the Ontario Institute for Cancer Research.

Doctors and hospital-based researchers were urged to take a more business-like approach. "Investigators don't think of themselves as business people. ... But they are in business. They are not practising medicine when they are doing a trial," said Margaret Kerr, a Toronto lawyer who specializes in contract work.

The meeting, organized by the private-sector group Insight Information, a division of Incisive Media, was attended by about 85 people, including the speakers and representatives from contract research organizations, brandname pharmaceutical companies and government, as well as academic researchers and contract lawyers. It's not often that such a diverse group meets, several participants noted.

Some of the many challenges facing the clinical trial industry identified at the meeting include:
- A lack of basic information about the clinical trial landscape in Canada and a lack of cooperation among the various parties

- The increased complexity of documentation required for clinical trials, lack of standardization in contracts and among research ethics boards, as well as other bureaucratic and administration complications

- The public's lack of confidence in pharmaceutical companies and difficulties recruiting patients for trials (with the exception of trials for cancer drugs)

- Clinical trials that are unnecessarily complex and/or uninteresting to researchers, and the time and cost pressures resulting from drug firm financial realities.

Although pharmaceutical companies have increasingly outsourced the clinical trial business to contract research organizations, (these private sector organizations now run more clinical trials than universities or hospitals), the sector is "fragmented and diversified," said Carl Viel, president of PÔLE, an agency established to promote economic development in the Québec City metropolitan area.

Indeed, when PÔLE set out to map the presence and activities of contract research organizations in the Québec City area, it had to hire a student for

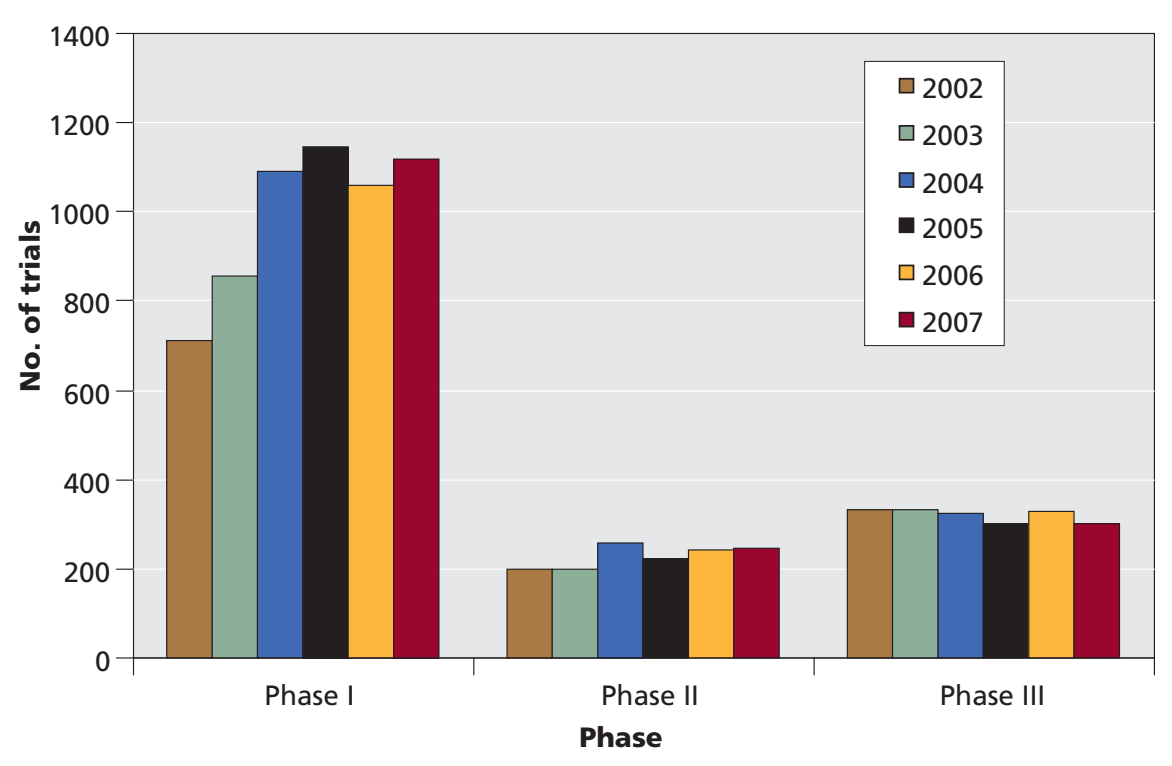

Figure 1: Canadian clinical trials, according to a Centerwatch analysis of Health

Canada's Therapeutics Directorate annual reports.

the task because the province could provide no information, Viel told the conference.

Meanwhile, little is known about where the organizations operate, because many are involved with running trials offshore. "Canadian companies have created thousands of jobs internationally."

Gervais said that government representatives have no idea about how much research is done in the private sector, "there is no structure to welcome more," and governments seem to deem the sector too small to warrant tracking.

Meanwhile, the academic clinical trial sector suffers from a similar lack of cooperation and information, speakers told the conference. Many Canadian hospitals and research institutions are aiming to improve their capacity to run clinical trials, and academic researchers are establishing and strengthening clinical trial specialty groups involving multiple sites.

But cooperation among hospitals "is the exception rather than the rule," said Phillips. Clinical trials are "grafted onto other hospital practices," he said, noting that when he offered to help design model systems for clinical trials, hospitals "were so burdened with patient care" that they couldn't spare the time to work with him. 
And within hospitals, individual site groups are "often autonomous empires with little oversight," Phillips added. When his institute established a database (www.ontariocancertrials.ca) of clinical trials "we thought it was for patients, but nurses use it to find out about trials in their own hospitals."

The current system typically works for well-known senior clinician scientists, who are sought out to conduct trials, but it can be hard for young scientists to get a foot in the door, he added.

Meanwhile, variations in contract language and in the requirements of research ethics boards pose problems for both hospitals and research institutes, speakers told the conference. The push to standardize contracts and to harmonize research ethics board approval standards is making slow progress.

The University of Toronto system has developed a template contract, and St. Michael's Hospital often settles on contract language with the sponsoring pharmaceutical company, said Michelle Moldofsky, a policy and legal adviser with the hospital's office of research administration.

But contract research organizations are "adding another party to the mix" and they "very rarely" agree on the sponsor's template, she said.

For Canada-wide trials, organizers must cope with differing provincial privacy legislation, and the increase in the number of foreign-sponsored trials is accompanied by language, cultural and legal challenges, she said.

"With the globalization of research, we have to reach through layers and layers of people," added Kerr. "We need a Batphone to the commissioner."

Foreign sponsors have also had some difficulty with the fact that Canadian physicians are not site employees but rather independent contractors, which affects the insurance provisions of contracts, the lawyer said.

For the Canadian subsidiaries of international pharmaceutical companies, difficulties can arise when trials are set by global colleagues so that subsidiaries are limited in their ability to influence contracts or protocols, several speakers said.
Kerr said another stumbling block for some sponsors is the University of Toronto's requirement that clinical trial sites report any adverse events suffered by patients to both Health Canada and the research ethics boards of other sites involved in a multisite trial.

Those adverse events may be becoming more common because of time pressures, suggested Janice Parente, president of ethica Clinical Research Inc. of Montreal, Quebec. Drugs are being withdrawn from the market because "the research was not done properly. ... Maybe we need to relax the time," she said. "We are making huge mistakes that affect humans."

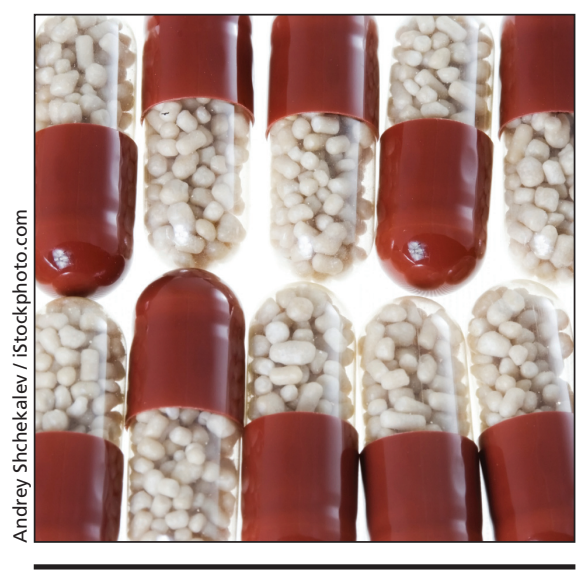

It is estimated that only $1 \%-3 \%$ of Canadians participate in clinical trials.

Heslegrave said that in protocol violations involving patients — for example, a pregnancy test not being undertaken on a trial participant - the excuse typically offered is that "there is no time."

Only $1 \%-3 \%$ of the eligible Canadian population participates in clinical trials, Wilson said. Speakers blamed the difficulty in recruiting patients (except for cancer patients) on widespread publicity about drug withdrawals, as well as a shift in the public's perception of drug companies - from discoverers of new medications to commodity providers.

This change in perception is likely fuelled by widespread direct-toconsumer advertising of prescription drugs (illegal in Canada but familiar to Canadians through United States television and magazines), said Murray Jensen, director of clinical and scientific affairs for ethica.
On the other hand, Pierre Geoffroy, a senior director with sanofi pasteur, suggested public confidence may have been undermined because advances in pharmacovigilance and post-market surveillance of drugs are leading to publicity about "post-market misadventures" that may have gone unidentified in the past.

To attract more doctors to clinical trials, the administrative burden on them must be lifted, said Wilson.

But part of the difficulty in recruiting physicians lies in the trials themselves, argued Muhammad Mamdani, director of the Applied Health Research Centre at the Li Ka Shing Knowledge Institute in Toronto. "I can't tell you the number of industry trials which are of no interest to doctors," he said.

Mamdani said the unnecessary complexity of trials is another stumbling block.

Heslegrave concurs. "At the REB [research ethic board] level, we see increasingly complex studies that try to do too much, that have one primary and lots of secondary objectives, and are not powered enough, so questions arise if these are even valid studies."

For Canada to "survive and prosper" with clinical trials, there must be more early cooperation on study design between academics and the pharmaceutical companies sponsoring the trials, and multicentre trials must be easier to initiate, said Phillips. "Companies can't just come to a CRO [contract research organization] and say, 'here it is, do it'."

In the future, clinical trials should answer not just whether or not a drug works for a specific population, but why, Phillips added. "Drugs should be based on science, not serendipity. The goal will be to design drugs targeted to patients and we need to recognize this change."

If contract research organizations want to be involved in high-impact clinical trials, they will have to build links with the academics, Phillips later said in an interview. "Everyone is groping with how to come to a mechanism to bring teams together." - Ann Silversides, CMAJ

DOI:10.1503/cmaj.081897 